\title{
Effective filtering analysis for non-Gaussian dynamic systems ${ }^{1}$
}

\author{
Yanjie Zhang ${ }^{a, 2}$, Huijie Qiao ${ }^{b, * 3}$ and Jinqiao Duan ${ }^{c, 4}$ \\ ${ }^{a}$ Center for Mathematical Sciences \& School of Mathematics and Statistics, \\ Huazhong University of Sciences and Technology, Wuhan 430074, China \\ ${ }^{b}$ School of Mathematics, \\ Southeast University, Nanjing, Jiangsu 211189, China \\ ${ }^{c}$ Department of Applied Mathematics, \\ Illinois Institute of Technology, Chicago, IL 60616, USA
}

\begin{abstract}
This work is about a slow-fast data assimilation system under non-Gaussian noisy fluctuations. Firstly, we show the existence of a random invariant manifold for a stochastic dynamical system with non-Gaussian noise and two-time scales. Secondly, we obtain a low dimensional reduction of this system via a random invariant manifold. Thirdly, we prove that the low dimensional filter on the random invariant manifold approximates the original filter, in a probabilistic sense.

Keywords: Random invariant manifold, $\alpha$-stable noise, Zakai equation, data assimilation, non-Gaussian noise.
\end{abstract}

\section{Introduction}

Data assimilation is a procedure to extract system state information with help of noisy observations [1, 2, 3]. The state evolution is often non-Gaussian (in particular, Lévy type) in complex systems. Data assimilation systems under

\footnotetext{
${ }^{1}$ AMS Subject Classification(2010): 60H10, 37D10, 70K70. This work was partly supported by the NSF grant 1620449, and NSFC grants 11371352, 11531006 and 11771449.

2 zhangyanjie2011@163.com .

${ }^{3}$ corresponding author: hjqiaogean@seu.edu.cn.

${ }^{4}$ duan@iit.edu
} 
non-Gaussian noisy fluctuations have been recently considered by us and other authors [4, 5, 6, 7]. It is also desirable to consider data assimilation when the system evolution is under Lévy motions with two-time scales.

Stochastic dynamical systems with non-Gaussian noise with two-time scales arise widely in mathematical modeling [6, 8, 9, 20]. Treating stochastic differential equations (SDEs) with two-time scales, Khasminskii and Yin [10] developed a stochastic averaging principle that enables to average out the fast varying variables. Recently, we have further showed that the averaged, low dimensional formal filter approximates the original filter, by examining the corresponding Zakai stochastic partial differential equations [11].

The theory of random invariant manifolds provides a geometric approach for eliminating the fast variables by a fixed point technique [20, 12]. For slow-fast SDEs with Gaussian noise on the long time scales, qualitative analysis for the behavior of random invariant manifold can be found in Wang and Roberts [13]. Filtering problems on a random invariant manifold driven by Gaussian noise have been studied [14]. In this present paper, our goal is to investigate filtering for stochastic differential equations with $\alpha$-stable Lévy noise and two-time scales, when we can only observe the slow component. Firstly, we show the existence of a random invariant manifold for a stochastic dynamical system driven by $\alpha$-stable noise with two-time scales. Secondly, we obtain its low dimensional reduction via the random invariant manifold. Thirdly, we prove that the low dimensional filter on the random invariant manifold approximates the original filter, as the scale parameter tends to zero.

This paper is organized as follows. In Section 2, we recall basic concepts about symmetric $\alpha$-stable Lévy process and random dynamical systems. Section 3 is devoted to the existence of a random invariant manifold for a slow-fast stochastic system with $\alpha$-stable Lévy process. In Section 4 , we present a nonlinear filtering problem this slow-fast system, and prove that the low dimensional filter approximates the original filter on the random invariant manifold. Finally, we end the paper with a summary on our results and some discussions.

The following convention will be used throughout the paper: $C$ denotes 
different positive constants whose values may change from one place to another; $\mathcal{C}\left(\mathbb{R}^{n}\right)$ is the set of all real-valued continuous functions on $\mathbb{R}^{n}$; we denote by $\mathcal{C}_{b}^{1}\left(\mathbb{R}^{n}\right)$ the set of all continuous functions on $\mathbb{R}^{n}$, with first-order derivatives are uniformly bounded. For $\phi \in \mathcal{C}_{b}^{1}\left(\mathbb{R}^{n}\right)$, we introduce the following norm

$$
\|\phi\|=\max _{x \in \mathbb{R}^{n}}|\phi(x)|+\max _{x \in \mathbb{R}^{n}}|\nabla \phi(x)|
$$

where $\nabla$ represents the gradient operator.

Let $\operatorname{Lip}\left(\mathbb{R}^{m}, \mathbb{R}^{n}\right)$ denote the set of globally Lipschitz continuous functions $\gamma$ mapping $\mathbb{R}^{m}$ into $\mathbb{R}^{n}$ with the following semi-norm

$$
\|\gamma\|_{L i p}:=\sup _{y_{1} \neq y_{2} \in \mathbb{R}^{m}} \frac{\left|\gamma\left(y_{1}\right)-\gamma\left(y_{2}\right)\right|}{\left|y_{1}-y_{2}\right|}<\infty
$$

and $\mathcal{L}$ the subset of $\operatorname{Lip}\left(\mathbb{R}^{m}, \mathbb{R}^{n}\right)$ consisting of bounded functions with the following norm

$$
\|\gamma\|_{\infty}:=\sup _{\tilde{y} \in \mathbb{R}^{m}}|\gamma(\tilde{y})| .
$$

The set $\mathcal{L}_{\kappa} \subseteq \mathcal{L}$ whose each element $\gamma$ satisfies $\|\gamma\|_{L i p} \leq \kappa$.

\section{Preliminaries}

In this section, we recall some basic definitions for Lévy motions (or Lévy processes) and random dynamical systems.

2.1. Symmetric $\alpha$-stable Lévy processes (see [15, 16])

Definition 1. A stochastic process $L_{t}$ is a Lévy process if

(1) $L_{0}=0$ (a.s.);

(2) $L_{t}$ has independent increments and stationary increments; and

(3) $L_{t}$ has stochastically continuous sample paths, i.e., for every $s \geq 0, L(t) \rightarrow$ $L(s)$ in probability, as $t \rightarrow s$.

A Lévy process $L_{t}$ taking values in $\mathbb{R}^{n}$ is characterized by a drift vector $b \in \mathbb{R}^{n}$, an $n \times n$ non-negative-definite, symmetric covariance matrix $Q$ and a 
Borel measure $\nu$ defined on $\mathbb{R}^{n} \backslash\{0\}$. We call $(b, Q, \nu)$ the generating triplet of the Lévy motions $L_{t}$. Moreover, we have the Lévy-Itô decomposition for $L_{t}$ as follows:

$$
L_{t}=b t+B_{Q}(t)+\int_{|y|<1} y \tilde{N}(t, d y)+\int_{|y| \geq 1} y N(t, d y)
$$

where $N(d t, d y)$ is the Poisson random measure, $\widetilde{N}(d t, d y)=N(d t, d y)-\nu(d x) d t$ is the compensated Poisson random measure, $\nu(A)=\mathbb{E} N(1, A)$ is the jump measure, and $B_{Q}(t)$ is an independent standard $n$-dimensional Brownian motion. The characteristic function of $L_{t}$ is given by

$$
\mathbb{E}\left[\exp \left(\mathrm{i}\left\langle u, L_{t}\right\rangle\right)\right]=\exp (t \rho(u)), \quad u \in \mathbb{R}^{n},
$$

where the function $\rho: \mathbb{R}^{n} \rightarrow \mathbb{C}$ is the characteristic exponent

$$
\rho(u)=\mathrm{i}\langle u, b\rangle-\frac{1}{2}\langle u, Q u\rangle+\int_{\mathbb{R}^{n} \backslash\{0\}}\left(e^{\mathrm{i}\langle u, z\rangle}-1-\mathrm{i}\langle u, z\rangle I_{\{|z|<1\}}\right) \nu(d z) .
$$

The Borel measure $\nu$ is called the jump measure. Here $\langle\cdot, \cdot\rangle$ denotes the scalar product in $\mathbb{R}^{n}$.

Definition 2. For $\alpha \in(0,2)$, a n-dimensional symmetric $\alpha$-stable process $L_{t}^{\alpha}$ is a Lévy process with characteristic exponent $\rho$

$$
\rho(u)=-C_{1}(n, \alpha)|u|^{\alpha}, \text { for } u \in \mathbb{R}^{n}
$$

with $C_{1}(n, \alpha):=\pi^{-\frac{1}{2}} \Gamma((1+\alpha) / 2) \Gamma(n / 2) / \Gamma((n+\alpha) / 2)$.

For a $n$-dimensional symmetric $\alpha$-stable Lévy process, the diffusion matrix $Q=0$, the drift vector $b=0$, and the Lévy measure $\nu$ is given by

$$
\nu(d u)=\frac{C_{2}(n, \alpha)}{|u|^{n+\alpha}} d u,
$$

where $C_{2}(n, \alpha):=\alpha \Gamma((n+\alpha) / 2) /\left(2^{1-\alpha} \pi^{n / 2} \Gamma(1-\alpha / 2)\right)$. 


\subsection{Random dynamical systems (see [1\}])}

Definition 3. Let $(H, \mathscr{B}(H))$ be a measurable space. A mapping

$$
\phi: \mathbb{R} \times \Omega \times H \mapsto H, \quad(t, \omega, x) \mapsto \phi(t, \omega, x)
$$

with the following properties is called a measurable random dynamical system $(R D S)$, if it is jointly $\mathscr{B}(\mathbb{R}) \otimes \mathscr{F} \otimes \mathscr{B}(H) / \mathscr{B}(H)$ measurable and satisfies the cocycle property:

$$
\begin{gathered}
\phi(0, \omega, \cdot)=\operatorname{id}_{H}, \text { for each } \omega \in \Omega ; \\
\phi(t+s, \omega, \cdot)=\phi\left(t, \theta_{s} \omega, \phi(s, \omega, \cdot)\right), \text { for each } s, t \in \mathbb{R}, \omega \in \Omega .
\end{gathered}
$$

Here we present a simple example to illustrate the abstract definitions of measurable random dynamical system.

Example 1. Consider a scalar linear stochastic differential equation

$$
d X_{t}=2 d B_{t}, X_{0}=x
$$

The solution mapping is $\phi(t, \omega, x)=2 B_{t}(\omega)+x$. Note that $\phi(0, \omega, x)=x$, and $\phi(t+s, \omega, x)=2 B_{t+s}(\omega)+x=\phi\left(t, \theta_{s}(\omega), \varphi(s, \omega, x)\right)$.

\subsection{Random invariant manifolds (see [1] ] ])}

Let $\phi$ be a random dynamical system on the normed space $(H, \mathscr{B}(H))$. We introduce a random invariant manifold with respect to $\phi$.

A family of nonempty closed set $\mathcal{M}=\{\mathcal{M}(\omega)\}_{\omega \in \Omega}$ is called a random set if for every $z \in H$, the mapping

$$
\Omega \ni \omega \mapsto \operatorname{dist}(z, \mathcal{M}(\omega)):=\inf _{z^{\prime} \in \mathcal{M}(\omega)}\left\|z-z^{\prime}\right\|_{H}
$$

is measurable. Moreover, $\mathcal{M}$ is called a positively invariant set with respect to the random dynamical system $\phi$ if

$$
\phi(t, \omega, \mathcal{M}(\omega)) \subseteq \mathcal{M}\left(\theta_{t} \omega\right), \text { for } t \in \mathbb{R}^{+}, \omega \in \Omega .
$$


In the sequel, we suppose $H=\mathbb{R}^{n} \times \mathbb{R}^{m}$ and consider random sets defined by a Lipschitz continuous graph. Let

$$
\begin{gathered}
\gamma: \Omega \times \mathbb{R}^{m} \rightarrow \mathbb{R}^{n} \\
(\omega, y) \mapsto \gamma(\omega, y)
\end{gathered}
$$

be a function such that $\gamma(\omega, y)$ is globally Lipschitzian in $y$ and for any $y \in \mathbb{R}^{m}$ and that the mapping $\omega \mapsto \gamma(\omega, y)$ is a random variable. Then $\mathcal{M}(\omega):=$ $\left\{(\gamma(\omega, y), y): y \in \mathbb{R}^{m}\right\}$ is a random set (see [19]). The invariant random set $\mathcal{M}(\omega)$ is called a Lipschitz random invariant manifold.

\section{Existence of a random invariant manifold}

In this section, we will present the reduction method for a slow-fast stochastic dynamical system with $\alpha$-stable Lévy process. Here we only present main results which will be used in the next section. The detailed illustrations will be presented in the Appendix (Section 6).

Let $\mathcal{D}_{0}\left(\mathbb{R}, \mathbb{R}^{n}\right)$ be the space of càdlàg functions starting at 0 given by

$\mathcal{D}_{0}=\left\{\omega:\right.$ for $\forall t \in \mathbb{R}, \lim _{s \uparrow t} \omega(s)=\omega(t-), \lim _{s \downarrow t} \omega(s)=\omega(t)$ exist and $\left.\omega(0)=0\right\}$.

For functions $\omega_{1}, \omega_{2} \in \mathcal{D}_{0}, \mathrm{~d}_{\mathbb{R}}\left(\omega_{1}, \omega_{2}\right)$ is given by

$$
\begin{array}{r}
\mathrm{d}_{\mathbb{R}}\left(\omega_{1}, \omega_{2}\right)=\inf \left\{\varepsilon>0:\left|\omega_{1}(t)-\omega_{2}(\lambda t)\right| \leq \varepsilon,\left|\ln \frac{\arctan (\lambda t)-\arctan (\lambda s)}{\arctan (t)-\arctan (s)}\right| \leq \varepsilon\right. \\
\text { for every } \left.t, s \in \mathbb{R} \text { and some } \lambda \in \mathbb{R}^{\mathbb{R}}\right\},
\end{array}
$$

where

$\mathbb{R}^{\mathbb{R}}=\left\{\lambda: \mathbb{R} \rightarrow \mathbb{R} ; \lambda\right.$ is injective increasing, $\left.\lim _{t \rightarrow-\infty} \lambda(t)=-\infty, \lim _{t \rightarrow \infty} \lambda(t)=\infty\right\}$

Denote by $\mathscr{B}\left(\mathcal{D}_{0}\right)$ the associated Borel $\sigma$-algebra generated by $\mathcal{D}_{0}$, then $\left(\mathcal{D}_{0}, \mathscr{B}\left(\mathcal{D}_{0}\right)\right)$ is a separable metric space. The probability measure $\mathbb{P}$ is generated 
by $\mathbb{P}\left(\mathcal{D}_{0} \cap A\right):=\mathbb{P}_{\mathbb{R}}(A)$ for each $A \in \mathscr{B}(\mathbb{R})$. The Wiener shift

$$
\begin{aligned}
& \theta: \mathbb{R} \times \mathcal{D}_{0} \rightarrow \mathcal{D}_{0}, \\
& \theta_{t} \omega(\cdot) \mapsto \omega(\cdot+t)-\omega(t) .
\end{aligned}
$$

is a Carathéodory function. Obviously, the Wiener shift $\theta$ is jointly measurable. The probability measure $\mathbb{P}$ is $\theta$ - invariant and the metric dynamical system

$$
\left(\mathcal{D}_{0}, \mathscr{B}\left(\mathcal{D}_{0}\right), \mathbb{P},\left(\theta_{t}\right)_{t \in \mathbb{R}}\right)
$$

is ergodic (see [12]).

Define $\left(\Omega^{1}, \mathcal{F}^{1}, \mathbb{P}^{1}, \theta_{t}^{1}\right)=\left(\mathcal{D}_{0}, \mathscr{B}\left(\mathcal{D}_{0}\right), \mathbb{P},\left(\theta_{t}\right)_{t \in \mathbb{R}}\right)$. Then $\left(\Omega^{1}, \mathcal{F}^{1}, \mathbb{P}^{1}, \theta_{t}^{1}\right)$ is a metric dynamical system. Similarly, we define $\Omega^{2}, \mathcal{F}^{2}, \mathbb{P}^{2}, \theta_{t}^{2}$. Then $\left(\Omega^{2}, \mathcal{F}^{2}, \mathbb{P}^{2}, \theta_{t}^{2}\right)$ is another metric dynamical system. Introduce

$$
\Omega:=\Omega^{1} \times \Omega^{2}, \mathcal{F}:=\mathcal{F}^{1} \times \mathcal{F}^{2}, \mathbb{P}:=\mathbb{P}^{1} \times \mathbb{P}^{2}, \theta_{t}:=\theta_{t}^{1} \times \theta_{t}^{2},
$$

and then $\left(\Omega, \mathcal{F}, \mathbb{P}, \theta_{t}\right)$ is a metric dynamical system.

In this paper, we consider the following fast-slow stochastic dynamical system

$$
\left\{\begin{array}{l}
d X_{t}^{\varepsilon}=\frac{1}{\varepsilon} A X_{t}^{\varepsilon} d t+\frac{1}{\varepsilon} F\left(X_{t}^{\varepsilon}, Y_{t}^{\varepsilon}\right) d t+\frac{\sigma_{1}}{\varepsilon^{\frac{1}{\alpha_{1}}}} d L_{1}^{\alpha_{1}}, \quad X_{0}^{\varepsilon}=x \in \mathbb{R}^{n} \\
d Y_{t}^{\varepsilon}=B Y_{t}^{\varepsilon} d t+G\left(X_{t}^{\varepsilon}, Y_{t}^{\varepsilon}\right) d t+\sigma_{2} d L_{2}^{\alpha_{2}}, \quad Y_{0}^{\varepsilon}=y \in \mathbb{R}^{m}
\end{array}\right.
$$

Here $\left(X_{t}^{\varepsilon}, Y_{t}^{\varepsilon}\right)$ is an $\mathbb{R}^{n} \times \mathbb{R}^{m}$-valued signal process which contains the fast and slow components, respectively. The interaction functions $F: \mathbb{R}^{n} \times \mathbb{R}^{m} \rightarrow \mathbb{R}^{n}$ and $G: \mathbb{R}^{n} \times \mathbb{R}^{m} \rightarrow \mathbb{R}^{m}$ are Borel measurable respectively. The constant matrixes $A$ and $B$ are $\mathbb{R}^{n} \times \mathbb{R}^{n}$ and $\mathbb{R}^{m} \times \mathbb{R}^{m}$ respectively. Both $\sigma_{1}$ and $\sigma_{2}$ are nonzero real noise intensities. The non-Gaussian processes $L_{1}^{\alpha_{1}}, L_{2}^{\alpha_{2}}$ (with $1<\alpha_{1}, \alpha_{2}<2$ ) are independent symmetric $\alpha$-stable Lévy processes with triplets $\left(0,0, \nu_{1}\right)$ and $\left(0,0, \nu_{2}\right)$, respectively. The small parameter $\varepsilon(0<\varepsilon \ll 1)$ is the ratio of the two time scales. We make the following assumptions on the signal system. 
Hypothesis H.1 There exists a constant $M_{A}>0$ such that

$$
(A x, x) \leq-M_{A}|x|^{2}, \text { for all } x \in \mathbb{R}^{n} .
$$

For every $y \in \mathbb{R}^{m},|B y| \leq\|B\||y|$, where $\|B\|$ standing for the norm of the matrix $B$, and $-B$ has no eigenvalue on the imaginary axis.

Hypothesis H.2 The functions $F, G$ satisfy the global Lipschitz conditions and sublinear growth condition, i.e., there exists positive constants $L$ and $K$ such that for all $x_{i} \in \mathbb{R}^{n}, y_{i} \in \mathbb{R}^{m}, i=1,2$ or $x \in \mathbb{R}^{n}, y \in \mathbb{R}^{m}$, we have

$$
\begin{aligned}
& \left|F\left(x_{1}, y_{1}\right)-F\left(x_{2}, y_{2}\right)\right| \leq L\left(\left|x_{1}-x_{2}\right|+\left|y_{1}-y_{2}\right|\right), \\
& \left|G\left(x_{1}, y_{1}\right)-G\left(x_{2}, y_{2}\right)\right| \leq L\left(\left|x_{1}-x_{2}\right|+\left|y_{1}-y_{2}\right|\right),
\end{aligned}
$$

and

$$
|F(x, y)|^{2}+\mid G\left(x,\left.y\right|^{2} \leq K\left[1+|x|^{2}+|y|^{2}\right] .\right.
$$

Hypothesis H.3 $M_{A}>L$.

Hypothesis H.4 The function $F$ and $G$ are uniformly bounded, i.e.,

$$
\begin{array}{r}
\sup _{(x, y) \in \mathbb{R}^{n} \times \mathbb{R}^{m}}|F(x, y)|<\infty, \\
\sup _{(x, y) \in \mathbb{R}^{n} \times \mathbb{R}^{m}}|G(x, y)|<\infty .
\end{array}
$$

Remark 1. Hypothesis $\mathbf{H . 3}$ can be interpreted as a spectral gap condition.

Remark 2. Under the assumptions $\mathbf{H . 2}$ and H.4, the system (3.4) has a global unique solution $\left(X^{\varepsilon}(t), Y^{\varepsilon}(t)\right)$, with a given initial value $(x(0), y(0))$.

Introduce the following two auxiliary systems

$$
\begin{aligned}
d \xi^{\varepsilon}(t) & =\frac{1}{\varepsilon} A \xi^{\varepsilon}(t) d t+\frac{\sigma_{1}}{\varepsilon^{\frac{1}{\alpha_{1}}}} d L_{t}^{\alpha_{1}}\left(\omega_{1}\right), \quad \xi(0)=\xi_{0}, \\
d \eta(t) & =B \eta(t) d t+\sigma_{2} d L_{t}^{\alpha_{2}}\left(\omega_{2}\right), \quad \eta(0)=\eta_{0} .
\end{aligned}
$$

By [Appendix, Lemma 1], there exist two random variables $\xi^{\varepsilon}(t)$ and $\eta(t)$ such 
that $\xi^{1, \frac{1}{\varepsilon}}\left(\theta_{t}^{1} \omega_{1}\right)$ and $\eta\left(\theta_{t}^{2} \omega_{2}\right)$ solve the equations (3.6). Set

$$
\left(\begin{array}{c}
\bar{x}^{\varepsilon}(t) \\
\bar{y}^{\varepsilon}(t)
\end{array}\right):=\left(\begin{array}{c}
X^{\varepsilon}(t)-\xi^{1, \frac{1}{\varepsilon}}\left(\theta_{t}^{1} \omega_{1}\right) \\
Y^{\varepsilon}(t)-\eta\left(\theta_{t}^{2} \omega_{2}\right)
\end{array}\right)
$$

then $\left(\bar{x}^{\varepsilon}(t), \bar{y}^{\varepsilon}(t)\right)$ satisfies the following equations

$$
\left\{\begin{array}{l}
\dot{\bar{x}}^{\varepsilon}(t)=\frac{1}{\varepsilon} A \bar{x}^{\varepsilon}(t)+\frac{1}{\varepsilon} F\left(\bar{x}^{\varepsilon}(t)+\xi^{1, \frac{1}{\varepsilon}}\left(\theta_{t}^{1} \omega_{1}\right), \bar{y}^{\varepsilon}(t)+\eta\left(\theta_{t}^{2} \omega_{2}\right)\right) \\
\dot{\bar{y}}^{\varepsilon}(t)=B \bar{y}^{\varepsilon}(t)+G\left(\bar{x}^{\varepsilon}(t)+\xi^{1, \frac{1}{\varepsilon}}\left(\theta_{t}^{1} \omega_{1}\right), \bar{y}^{\varepsilon}(t)+\eta\left(\theta_{t}^{2} \omega_{2}\right)\right)
\end{array}\right.
$$

The scaling $t \rightarrow \varepsilon t$ in (3.8) yields

$$
\left\{\begin{array}{l}
\frac{d x}{d t}=A x+F\left(x+\xi^{1, \frac{1}{\varepsilon}}\left(\theta_{\varepsilon t}^{1} \omega_{1}\right), y+\eta\left(\theta_{\varepsilon t}^{2} \omega_{2}\right)\right), \\
\frac{d y}{d t}=\varepsilon B y+\varepsilon G\left(x+\xi^{1, \frac{1}{\varepsilon}}\left(\theta_{\varepsilon t}^{1} \omega_{1}\right), y+\eta\left(\theta_{\varepsilon t}^{2} \omega_{2}\right)\right) .
\end{array}\right.
$$

If we now replace $\xi^{1, \frac{1}{\varepsilon}}\left(\theta_{\varepsilon t}^{1} \omega_{1}\right)$ by $\xi^{1,1}\left(\theta_{t}^{1} \omega_{1}\right)$ that has the same distribution, then we obtain a system of the following form

$$
\left\{\begin{array}{l}
\frac{d \bar{X}^{\varepsilon}(t)}{d t}=A \bar{X}^{\varepsilon}(t)+F\left(\bar{X}^{\varepsilon}(t)+\xi^{1,1}\left(\theta_{t}^{1} \omega_{1}\right), \bar{Y}^{\varepsilon}(t)+\eta\left(\theta_{\varepsilon t}^{2} \omega_{2}\right)\right) \\
\frac{d \bar{Y}^{\varepsilon}(t)}{d t}=\varepsilon B \bar{Y}^{\varepsilon}(t)+\varepsilon G\left(\bar{X}^{\varepsilon}(t)+\xi^{1,1}\left(\theta_{t}^{1} \omega_{1}\right), \bar{Y}^{\varepsilon}(t)+\eta\left(\theta_{\varepsilon t}^{2} \omega_{2}\right)\right) .
\end{array}\right.
$$

The system (3.10) has a unique global solution $\phi^{\varepsilon}\left(t, \omega,\left(x_{0}, y_{0}\right)\right)$ for any initial condition $\left(x_{0}, y_{0}\right) \in \mathbb{R}^{n+m}$, the solution operator of the initial value problem to system (3.10) denoted by

$$
\phi^{\varepsilon}\left(t, \omega,\left(x_{0}, y_{0}\right)\right):=\left(\phi_{1}^{\varepsilon}\left(t, \omega,\left(x_{0}, y_{0}\right)\right), \phi_{2}^{\varepsilon}\left(t, \omega,\left(x_{0}, y_{0}\right)\right)\right)
$$

Let

$$
\left\{\begin{array}{l}
F\left(\theta_{t}^{\varepsilon} \omega, \bar{X}^{\varepsilon}, \bar{Y}^{\varepsilon}\right)=F\left(\bar{X}^{\varepsilon}(t)+\xi^{1,1}\left(\theta_{t}^{1} \omega_{1}\right), \bar{Y}^{\varepsilon}(t)+\eta\left(\theta_{\varepsilon t}^{2} \omega_{2}\right)\right) \\
G\left(\theta_{t}^{\varepsilon} \omega, \bar{X}^{\varepsilon}, \bar{Y}^{\varepsilon}\right)=G\left(\bar{X}^{\varepsilon}(t)+\xi^{1,1}\left(\theta_{t}^{1} \omega_{1}\right), \bar{Y}^{\varepsilon}(t)+\eta\left(\theta_{\varepsilon t}^{2} \omega_{2}\right)\right)
\end{array}\right.
$$


Then the equation (3.10) can be rewritten as

$$
\left\{\begin{array}{l}
\frac{d \bar{X}^{\varepsilon}(t)}{d t}=A \bar{X}^{\varepsilon}(t)+F\left(\theta_{t}^{\varepsilon} \omega, \bar{X}^{\varepsilon}, \bar{Y}^{\varepsilon}\right) \\
\frac{d \bar{Y}^{\varepsilon}(t)}{d t}=\varepsilon B \bar{Y}^{\varepsilon}(t)+\varepsilon G\left(\theta_{t}^{\varepsilon} \omega, \bar{X}^{\varepsilon}, \bar{Y}^{\varepsilon}\right) .
\end{array}\right.
$$

The following theorem indicates the random dynamical system defined by (3.12) has a random invariant manifold.

Theorem 1. Assue that the hypotheses (H.1)-(H.4) hold. Then for sufficiently small $\varepsilon$ and sufficiently large $T$, the random dynamical system defined by (3.12) has a random invariant manifold, with graph defined by $\gamma^{\varepsilon}(\omega)$.

Proof. Assume $\mathcal{A}^{\varepsilon}$ has a unique fixed point $\gamma^{\varepsilon}$, where $\mathcal{A}^{\varepsilon}$ is defined in the appendix (6.46). Hence replacing $\omega$ by $\theta_{T}^{\varepsilon} \omega$, we have

$$
\psi^{\varepsilon}\left(t, \omega, \gamma^{\varepsilon}\right)=\gamma^{\varepsilon}\left(\theta_{T}^{\varepsilon} \omega, \cdot\right)
$$

Therefore for $t>0$, we have

$$
\begin{aligned}
\psi^{\varepsilon}\left(t, \omega, \gamma^{\varepsilon}(\omega)\right) & =\psi^{\varepsilon}\left(t, \cdot, \mathcal{A}^{\varepsilon}\left(\gamma^{\varepsilon}(\cdot)\right)\right)(\omega) \\
& =\psi^{\varepsilon}\left(t, \varepsilon, \psi^{\varepsilon}\left(T, \theta_{-T}^{\varepsilon} \omega, \gamma^{\varepsilon}\left(\theta_{-T^{\varepsilon}}^{\omega}\right)\right)\right) \\
& =\psi^{\varepsilon}\left(t+T, \theta_{-T}^{\varepsilon} \omega, \gamma^{\varepsilon}\left(\theta_{-T}^{\varepsilon} \omega\right)\right) \\
& =\psi^{\varepsilon}\left(T, \theta_{-T+t}^{\varepsilon} \omega, \psi^{\varepsilon}\left(T, \theta_{-T}^{\varepsilon} \omega, \gamma^{\varepsilon}\left(\theta_{-T}^{\varepsilon} \omega\right)\right)\right) \\
& =\mathcal{A}^{\varepsilon}\left(\psi^{\varepsilon}\left(t, \theta_{-t^{\varepsilon}}^{\varepsilon}, \gamma^{\varepsilon}\left(\theta_{-t^{\varepsilon}}^{\varepsilon}\right)\right)\left(\theta_{t}^{\varepsilon} \omega\right)\right. \\
& =\gamma^{\varepsilon}\left(\theta_{t}^{\varepsilon}\right) .
\end{aligned}
$$

Therefore the random dynamical system (3.12) has a random invariant Lipschitz manifold defined by $\left\{\left(\gamma^{\varepsilon}(\omega, \tilde{y}), \tilde{y}\right)\right\}$.

Remark 3. Thus the systems derived from (3.8) has a random invariant manifold with the same graph.

Based on the relationship between (3.4) and (3.8), it holds that the system 
(3.4) has a random invariant manifold

$$
M^{\varepsilon}(\omega)=\left\{\gamma^{\varepsilon}(\omega, \widetilde{y})+\xi^{1, \frac{1}{\varepsilon}}\left(\omega_{1}\right), \widetilde{y}+\eta\left(\omega_{2}\right)\right\}
$$

Therefore we can get the following reduction system which describes the behavior for system (3.4).

Theorem 2. Assume that the assumptions (H.1)-(H.4) hold. Then for any solution $z^{\epsilon}(t)=\left(X_{t}^{\epsilon}, Y_{t}^{\epsilon}\right)$ to system (3.4) with initial data $z^{\epsilon}(0)=\left(X_{0}^{\epsilon}, Y_{0}^{\epsilon}\right)$, there exists the following reduced low dimensional systems on the random invariant manifold

$$
\left\{\begin{aligned}
d y_{t}^{\varepsilon} & =B y_{t}^{\varepsilon} d t+G\left(x_{t}^{\varepsilon}, y_{t}^{\varepsilon}\right) d t+\sigma_{2} d L_{2}^{\alpha_{2}}, \\
x_{t}^{\varepsilon} & =\gamma^{\varepsilon}\left(\theta_{t}^{\varepsilon} \omega, y_{t}^{\varepsilon}-\eta\left(\theta_{t}^{2} \omega_{2}\right)\right)+\xi^{1, \frac{1}{\varepsilon}}\left(\theta_{t}^{1} \omega_{1}\right),
\end{aligned}\right.
$$

such that for sufficiently small $\varepsilon$, sufficiently large $t$ and some positive constant $\tilde{\kappa}$, we have

$$
\left\|z^{\varepsilon}(t, \omega)-\widetilde{z}^{\varepsilon}(t, \omega)\right\|_{\infty} \leq C_{\varepsilon, \tilde{\kappa}} e^{-\frac{\tilde{\kappa}}{\varepsilon} t}\left\|z^{\varepsilon}(0, \omega)-\widetilde{z}^{\varepsilon}(0, \omega)\right\|_{\infty},
$$

where $\widetilde{z}^{\epsilon}(t)=\left(x_{t}^{\epsilon}, y_{t}^{\epsilon}\right)$ is the solution of the low dimensional system (3.16) with initial data $\widetilde{z}^{\epsilon}(0)=\left(x_{0}^{\epsilon}, y_{0}^{\epsilon}\right)$.

Proof. Lemma 8 from appendix tells us that the system (3.8) has an exponentially tracking manifold, then so has the system (3.4).

\section{Approximation analysis for nonlinear filter}

In this section, we study the nonlinear filtering problem on the random invariant manifold. For $T>0$, an observation system is given by

$$
d Z_{t}^{\varepsilon}=h\left(Y_{t}^{\varepsilon}\right) d t+d W_{t}, \quad t \in[0, T]
$$

where $W_{t}$ is a standard Brownian motion independent of $L_{t}^{\alpha_{1}}$ and $L_{t}^{\alpha_{2}}$. In practical applications, we can only observe the slow component $Y_{t}^{\varepsilon}$. Let $(\Omega, \mathscr{F}, \mathbb{P})$ be 
a probability space together with a filtration $\left(\mathscr{F}_{t}\right)_{t \geq 0}$ which satisfies the usual conditions. For the observation system $Z_{t}^{\varepsilon}$, we make the following additional hypothesis.

Hypothesis H.5 The sensor function $h$ is bounded and Lipschitz continuous in $x$, with Lipschitz constant denoted by $\|h\|_{\text {Lip }}$.

Let

$$
\mathcal{Z}_{t}=\sigma\left(Z_{s}^{\varepsilon}: 0 \leq s \leq t\right) \vee \mathcal{N}
$$

where $\mathcal{N}$ is the collection of all $\mathbb{P}$-negligible sets of $(\Omega, \mathscr{F})$.

By the Girsanov's change of measure theorem, we obtain a new probability measure $\widetilde{\mathbb{P}}$, such that the observation $Z_{t}^{\varepsilon}$ becomes $\widetilde{\mathbb{P}}$-independent of the signal variables $\left(X_{t}^{\varepsilon}, Y_{t}^{\varepsilon}\right)$. In fact, this can be done through

$$
\left.\frac{d \widetilde{\mathbb{P}}}{d \mathbb{P}}\right|_{\mathscr{F}_{t}}=\exp \left(-\sum_{i=1}^{m} \int_{0}^{t} h^{i}\left(Y_{s}^{\varepsilon}\right) d W_{s}^{i}-\frac{1}{2} \sum_{i=1}^{m} \int_{0}^{t} h^{i}\left(Y_{s}^{\varepsilon}\right)^{2} d s\right)=:\left(\mathbb{R}_{t}^{\varepsilon}\right)^{-1}
$$

Hence we have

$$
\mathbb{R}_{t}^{\varepsilon}=\left.\frac{d \mathbb{P}}{d \widetilde{\mathbb{P}}}\right|_{\mathscr{F}_{t}}=\exp \left(\sum_{i=1}^{m} \int_{0}^{t} h^{i}\left(Y_{s}^{\varepsilon}\right) d\left(Z_{s}^{\varepsilon}\right)^{i}-\frac{1}{2} \sum_{i=1}^{m} \int_{0}^{t} h^{i}\left(Y_{s}^{\varepsilon}\right)^{2} d s\right) .
$$

Define

$$
\begin{aligned}
\rho_{t}^{\varepsilon}(\phi) & :=\widetilde{\mathbb{E}}\left[\phi\left(Y_{t}^{\varepsilon}\right) \mathbb{R}_{t}^{\varepsilon} \mid \mathcal{Z}_{t}\right], \\
\pi_{t}^{\varepsilon}(\phi) & :=\mathbb{E}\left[\phi\left(Y_{t}^{\varepsilon}\right) \mid \mathcal{Z}_{t}\right],
\end{aligned}
$$

where $\widetilde{\mathbb{E}}$ stands for the expectation under $\widetilde{\mathbb{P}}$ and $\phi \in \mathcal{C}_{b}^{1}\left(\mathbb{R}^{m}\right)$.

Then by the Kallianpur-Striebel formula, we have

$$
\pi_{t}^{\varepsilon}(\phi)=\frac{\rho_{t}^{\varepsilon}(\phi)}{\rho_{t}^{\varepsilon}(1)}
$$

Here $\pi_{t}^{\varepsilon}(\phi)$ is called the normalized filtering of $Y_{t}^{\varepsilon}$ with respect to $\mathcal{Z}_{t}$.

On the other hand, we can rewrite the reduced system (3.16) as

$$
d \widetilde{Y}_{t}^{\varepsilon}=B \widetilde{Y}_{t}^{\varepsilon} d t+\widetilde{G}^{\varepsilon}\left(\omega, \widetilde{Y}_{t}^{\varepsilon}\right) d t+\sigma_{2} d L_{2}^{\alpha_{2}}
$$


with

$$
\widetilde{G}^{\varepsilon}\left(\omega, \widetilde{Y}_{t}^{\varepsilon}\right):=G\left(\gamma^{\varepsilon}\left(\theta_{t}^{\varepsilon} \omega, \widetilde{Y}_{t}^{\varepsilon}-\eta\left(\theta_{t}^{2} \omega_{2}\right)\right)+\xi^{1, \frac{1}{\varepsilon}}\left(\theta_{t}^{1} \omega_{1}\right), \widetilde{Y}_{t}^{\varepsilon}\right)
$$

In the following, we are more interested in the reduced filtering problem with the actual observation, i.e., we will study the nonlinear filtering problem for the reduced system (4.7) with the actual observation $Z_{t}^{\varepsilon}$. Set

$$
\widetilde{\mathbb{R}}_{t}^{\varepsilon}:=\exp \left\{\int_{0}^{t} h\left(\widetilde{Y}_{s}^{\varepsilon}\right) d Z_{s}^{\varepsilon}-\frac{1}{2} \int_{0}^{t}\left|h\left(\widetilde{Y}_{s}^{\varepsilon}\right)\right|^{2} d s\right\}
$$

then $\widetilde{\mathbb{R}}_{t}^{\varepsilon}$ is an exponential martingale under $\widetilde{\mathbb{P}}$. Thus, we can define the "formally" non-normalized filtering for $\widetilde{Y}_{t}^{\varepsilon}$ by

$$
\widetilde{\rho}_{t}^{\varepsilon}(\phi):=\widetilde{\mathbb{E}}\left[\phi\left(\widetilde{Y}_{t}^{\varepsilon}\right) \widetilde{\mathbb{R}}_{t}^{\varepsilon} \mid \mathcal{Z}_{t}\right]
$$

And set

$$
\widetilde{\pi}_{t}^{\varepsilon}(\phi):=\frac{\widetilde{\rho}_{t}^{\varepsilon}(\phi)}{\widetilde{\rho}_{t}^{\varepsilon}(1)}
$$

then $\widetilde{\pi}_{t}^{\varepsilon}$ could be understood as the nonlinear filtering problem for $\widetilde{Y}_{t}^{\varepsilon}$ with respect to $\mathcal{Z}_{t}$.

Now, we are ready to state and prove the main result in this paper.

Theorem 3. Assume the hypotheses (H.1)-(H.5) hold. Then for $\forall \min \left(\frac{1}{4} \alpha_{1}, \frac{1}{4} \alpha_{2}\right)>p>0$, $\varepsilon$ sufficiently small and $t \in[0, T]$, there exists a positive constant $C$ such that for $\phi \in \mathcal{C}_{b}^{1}\left(\mathbb{R}^{m}\right)$

$$
\mathbb{E}\left|\pi_{t}^{\varepsilon}(\phi)-\widetilde{\pi}_{t}^{\varepsilon}(\phi)\right|^{p} \leq C|| \phi||^{p}\left(\mathbb{E}|| z^{\varepsilon}(0, \omega)-\widetilde{z}^{\varepsilon}(0, \omega) \|_{\infty}^{16 p}\right)^{\frac{1}{16}}\left(e^{\frac{-4 \tilde{\kappa} t p}{\varepsilon}}+\varepsilon\right)^{\frac{1}{4}}
$$

Proof. Step 1. As in [14], we have

$$
\mathbb{E}\left|\widetilde{\rho}_{t}^{\varepsilon}(1)\right|^{-p} \leq \exp \left\{\left(2 p^{2}+p+1\right) C T / 2\right\}, \quad t \in[0, T] .
$$


Step 2. For $\phi \in \mathcal{C}_{b}^{1}\left(\mathbb{R}^{m}\right)$, it follows from the Hölder inequality that

$$
\begin{aligned}
\mathbb{E}\left|\rho_{t}^{\varepsilon}(\phi)-\widetilde{\rho}_{t}^{\varepsilon}(\phi)\right|^{p} & =\widetilde{\mathbb{E}}\left[\left|\rho_{t}^{\varepsilon}(\phi)-\widetilde{\rho}_{t}^{\varepsilon}(\phi)\right|^{p} \mathbb{R}_{t}^{\varepsilon}\right] \\
& \leq\left(\widetilde{\mathbb{E}}\left[\left|\rho_{t}^{\varepsilon}(\phi)-\widetilde{\rho}_{t}^{\varepsilon}(\phi)\right|^{2 p}\right]\right)^{\frac{1}{2}}\left(\widetilde{\mathbb{E}}\left[\mathbb{R}_{t}^{\varepsilon}\right]^{2}\right)^{\frac{1}{2}} \\
& \leq \exp (C T / 2)\left(\widetilde{\mathbb{E}}\left[\left|\rho_{t}^{\varepsilon}(\phi)-\widetilde{\rho}_{t}^{\varepsilon}(\phi)\right|^{2 p}\right]\right)^{\frac{1}{2}}
\end{aligned}
$$

Step 3. Using the definitions of $\rho_{t}^{\varepsilon}(\phi)$ and $\widetilde{\rho}_{t}^{\varepsilon}(\phi)$, Jensen's inequality and Hölder inequality, we get

$$
\begin{aligned}
\widetilde{\mathbb{E}}\left|\rho_{t}^{\varepsilon}(\phi)-\widetilde{\rho}_{t}^{\varepsilon}(\phi)\right|^{2 p} & =\widetilde{\mathbb{E}}\left|\widetilde{\mathbb{E}}\left[\phi\left(Y_{t}^{\varepsilon}\right) \mathbb{R}_{t}^{\varepsilon} \mid \mathcal{Z}_{t}\right]-\widetilde{\mathbb{E}}\left[\phi\left(\widetilde{Y}_{t}^{\varepsilon}\right) \widetilde{\mathbb{R}}_{t}^{\varepsilon} \mid \mathcal{Z}_{t}\right]\right|^{2 p} \\
& \leq 2^{2 p-1} \widetilde{\mathbb{E}}\left[\left|\phi\left(Y_{t}^{\varepsilon}\right) \mathbb{R}_{t}^{\varepsilon}-\phi\left(\widetilde{Y}_{t}^{\varepsilon}\right) \mathbb{R}_{t}^{\varepsilon}\right|^{2 p}\right]+2^{2 p-1} \widetilde{\mathbb{E}}\left[\left|\phi\left(\widetilde{Y}_{t}^{\varepsilon}\right) \mathbb{R}_{t}^{\varepsilon}-\phi\left(\widetilde{Y}_{t}^{\varepsilon}\right) \widetilde{\mathbb{R}}_{t}^{\varepsilon}\right|^{2 p}\right] \\
& \leq\left. 2^{2 p-1}|| \phi\right|^{2 p} e^{p(4 p-1) C T}\left(\widetilde{\mathbb{E}}\left|Y_{t}^{\varepsilon}-\widetilde{Y}_{t}^{\varepsilon}\right|^{4 p}\right)^{\frac{1}{2}}+2^{2 p-1}\|\phi\|^{2 p} \widetilde{\mathbb{E}}\left[\left|\mathbb{R}_{t}^{\varepsilon}-\widetilde{\mathbb{R}}_{t}^{\varepsilon}\right|^{2 p}\right]
\end{aligned}
$$

Step 4. By the Itô's formula, BDG inequality and bounded property of $h$, we have

$$
\begin{aligned}
\widetilde{\mathbb{E}}\left[\left|\mathbb{R}_{t}^{\varepsilon}-\widetilde{\mathbb{R}}_{t}^{\varepsilon}\right|^{2 p}\right] & =\widetilde{\mathbb{E}}\left[\left|\int_{0}^{t}\left(\mathbb{R}_{s}^{\varepsilon} h\left(Y_{s}^{\varepsilon}\right)-\widetilde{\mathbb{R}}_{s}^{\varepsilon} h\left(\widetilde{Y}_{s}^{\varepsilon}\right)\right) d Z_{s}^{\varepsilon}\right|^{2 p}\right] \\
& \leq C \widetilde{\mathbb{E}}\left[\int_{0}^{t}\left|\mathbb{R}_{s}^{\varepsilon} h\left(Y_{s}^{\varepsilon}\right)-\widetilde{\mathbb{R}}_{s}^{\varepsilon} h\left(\widetilde{Y}_{s}^{\varepsilon}\right)\right|^{2} d s\right]^{p} \\
& \leq 2^{2 p-1} C T^{p-1} \int_{0}^{t} \widetilde{\mathbb{E}}\left|\mathbb{R}_{s}^{\varepsilon} h\left(Y_{s}^{\varepsilon}\right)-\mathbb{R}_{s}^{\varepsilon} h\left(\widetilde{Y}_{s}^{\varepsilon}\right)\right|^{2 p} d s \\
& +2^{2 p-1} C T^{p-1} \int_{0}^{t} \widetilde{\mathbb{E}}\left|\mathbb{R}_{s}^{\varepsilon} h\left(\widetilde{Y}_{s}^{\varepsilon}\right)-\widetilde{\mathbb{R}}_{s}^{\varepsilon} h\left(\widetilde{Y}_{s}^{\varepsilon}\right)\right|^{2 p} d s
\end{aligned}
$$

Then we have

$$
\widetilde{\mathbb{E}}\left[\left|\mathbb{R}_{t}^{\varepsilon}-\widetilde{\mathbb{R}}_{t}^{\varepsilon}\right|^{2 p}\right] \leq C\left(\widetilde{\mathbb{E}}|| z^{\varepsilon}(0)-\widetilde{z}^{\varepsilon}(0)||_{\infty}^{4 p}\right)^{\frac{1}{2}}+C \int_{0}^{t} \widetilde{\mathbb{E}}\left|\mathbb{R}_{s}^{\varepsilon}-\widetilde{\mathbb{R}}_{s}^{\varepsilon}\right|^{2 p} d s .
$$

Using Grönwall's inequality, we obtain that

$$
\widetilde{\mathbb{E}}\left[\left|\mathbb{R}_{t}^{\varepsilon}-\widetilde{\mathbb{R}}_{t}^{\varepsilon}\right|^{2 p}\right] \leq C\left(\widetilde{\mathbb{E}}\left\|z^{\varepsilon}(0)-\widetilde{z}^{\varepsilon}(0)\right\|_{\infty}^{4 p}\right)^{\frac{1}{2}}
$$


Step 5. Combing the results from Step 3 and Step 4, we get

$$
\mathbb{E}\left|\rho_{t}^{\varepsilon}(\phi)-\widetilde{\rho}_{t}^{\varepsilon}(\phi)\right|^{p} \leq C|| \phi||^{p}\left(\widetilde{\mathbb{E}}|| z^{\varepsilon}(0)-\widetilde{z}^{\varepsilon}(0)||_{\infty}^{4 p}\right)^{\frac{1}{4}}\left(e^{\frac{-2 \tilde{\kappa} t p}{\varepsilon}}+\varepsilon\right)^{\frac{1}{2}} .
$$

Step 6. Using the relationships between $\pi_{t}^{\varepsilon}(\phi), \widetilde{\pi}_{t}^{\varepsilon}(\phi)$ and $\rho_{t}^{\varepsilon}(\phi), \widetilde{\rho}_{t}^{\varepsilon}(\phi)$, we have

$$
\begin{aligned}
\mathbb{E}\left|\pi_{t}^{\varepsilon}(\phi)-\widetilde{\pi}_{t}^{\varepsilon}(\phi)\right|^{p} & =\mathbb{E}\left|\frac{\rho_{t}^{\varepsilon}(\phi)-\tilde{\rho}_{t}^{\varepsilon}(\phi)}{\tilde{\rho}_{t}^{\varepsilon}(1)}-\pi_{t}^{\varepsilon}(\phi) \frac{\rho_{t}^{\varepsilon}(1)-\tilde{\rho}_{t}^{\varepsilon}(1)}{\tilde{\rho}_{t}^{\varepsilon}(1)}\right|^{p} \\
& \leq 2^{p-1} \mathbb{E}\left|\frac{\rho_{t}^{\varepsilon}(\phi)-\tilde{\rho}_{t}^{\varepsilon}(\phi)}{\tilde{\rho}_{t}^{\varepsilon}(1)}\right|^{p}+2^{p-1} \mathbb{E}\left|\pi_{t}^{\varepsilon}(\phi) \frac{\rho_{t}^{\varepsilon}(1)-\tilde{\rho}_{t}^{\varepsilon}(1)}{\tilde{\rho}_{t}^{\varepsilon}(1)}\right|^{p} \\
& \leq 2^{p-1}\left(\mathbb{E}\left|\rho_{t}^{\varepsilon}(\phi)-\tilde{\rho}_{t}^{\varepsilon}(\phi)\right|^{2 p}\right)^{1 / 2}\left(\mathbb{E}\left|\tilde{\rho}_{t}^{\varepsilon}(1)\right|^{-2 p}\right)^{1 / 2} \\
& +2^{p-1}\|\phi\|^{p}\left(\mathbb{E}\left|\rho_{t}^{\varepsilon}(1)-\tilde{\rho}_{t}^{\varepsilon}(1)\right|^{2 p}\right)^{1 / 2}\left(\mathbb{E}\left|\tilde{\rho}_{t}^{\varepsilon}(1)\right|^{-2 p}\right)^{1 / 2} \\
& \leq C\|\phi\|^{p}\left(\mathbb{E}|| z^{\varepsilon}(0, \omega)-\widetilde{z}^{\varepsilon}(0, \omega) \|_{\infty}^{16 p}\right)^{\frac{1}{16}}\left(e^{\frac{-4 \tilde{\kappa} t p}{\varepsilon}}+\varepsilon\right)^{\frac{1}{4}} .
\end{aligned}
$$

Remark 4. For a stochastic differential equation with $\alpha$-stable Lévy noise, the existence for its $p$ moment requires $1<p<\alpha$.

\section{Conclusion}

We have presented a theoretical foundation for the development of effective filtering on a random invariant manifold in complex non-Gaussian multiscale systems. We can further extend this work to the case when the observation system is also driven by non-Gaussian noise. i.e.

$$
d Z_{t}^{\varepsilon}=h\left(Y_{t}^{\varepsilon}\right) d t+d L_{t}, \quad t \in[0, T]
$$

where $L_{t}$ is a standard Lévy process independent of $L_{t}^{\alpha_{1}}$ and $L_{t}^{\alpha_{2}}$.

Moreover, we can extend this work to slow-fast non-Gaussian stochastic partial differential equations, i.e., we consider the following fast-slow stochastic 
dynamical system

$$
\left\{\begin{array}{l}
d X_{t}^{\varepsilon}=\frac{1}{\varepsilon} A X_{t}^{\varepsilon} d t+\frac{1}{\varepsilon} F\left(X_{t}^{\varepsilon}, Y_{t}^{\varepsilon}\right) d t+\frac{\sigma_{1}}{\varepsilon^{\frac{1}{\alpha_{1}}}} d L_{1}^{\alpha_{1}} \\
d Y_{t}^{\varepsilon}=B Y_{t}^{\varepsilon} d t+G\left(X_{t}^{\varepsilon}, Y_{t}^{\varepsilon}\right) d t+\sigma_{2} d L_{2}^{\alpha_{2}} .
\end{array}\right.
$$

Here $\left(X_{t}^{\varepsilon}, Y_{t}^{\varepsilon}\right)$ is defined in two separable Hilbert space $\mathbb{H} \times \mathbb{H}$-valued signal process which represents the fast and slow components. The interaction functions $F: \mathbb{H} \times \mathbb{H} \rightarrow \mathbb{H}, G: \mathbb{H} \times \mathbb{H} \rightarrow \mathbb{H}$ are Borel measurable respectively. $A: \mathcal{D}(A) \subset H \mapsto H$ is a self-adjoint compact operator on $\mathbb{H}$ such that $-A$ has discrete spectrum $0<\mu_{1}<\mu_{2}<\cdots \mu_{k}<\cdots$ and $\lim _{k \rightarrow \infty} \mu_{k}=\infty$. $B: \mathcal{D}(B) \subset H \mapsto H$ is a linear unbounded operator on $\mathbb{H}$ such that $-B$ has discrete spectrum $0<\lambda_{1}<\lambda_{2}<\cdots \lambda_{k}<\cdots$ and $\lim _{k \rightarrow \infty} \lambda_{k}=\infty$. Both $\sigma_{1}$ and $\sigma_{2}$ are nonzero real noise intensities. The parameter $\varepsilon$ is the ratio of the slow time scale to the fast time scale. Non-Gaussian processes $L_{1}^{\alpha_{1}}, L_{2}^{\alpha_{2}}$ (with $\left.1<\alpha_{1}, \alpha_{2}<2\right)$ are a cylindrical $\alpha_{1}$-stable process and $\alpha_{2}$ stable process defined by the orthogonal expansion,respectively,

$$
\begin{aligned}
L_{1}^{\alpha_{1}}: & =\sum_{k=1}^{\infty} \beta_{k} L_{k}(t) e_{k}, \\
L_{2}^{\alpha_{2}}: & =\sum_{k=1}^{\infty} q_{k} Z_{k}(t) e_{k},
\end{aligned}
$$

where $\left\{e_{k}\right\}_{k \geq 1}$ is an orthonormal basis of $H,\left\{L_{k}(t)\right\}_{k \geq 1}$ and $\left\{Z_{k}(t)\right\}_{k \geq 1}$ are sequences of independent and identically distributed real-value symmetric $\alpha_{1^{-}}$ stable processes and $\alpha_{2}$-stable processes defined on the stochastic basis $\left(\Omega, \mathcal{F}, \mathcal{F}_{t}, \mathbb{P}\right)$, respectively, and $\beta_{k}, q_{k}>0$ for each $k \geq 1$.

\section{Appendix}

In this Appendix, we present materials about the existence of a random invariant manifold. Firstly, we recall the basic definition of stationary solution.

Definition 4. A random variable $\omega \mapsto y(\omega)$ with values in $H$ is called a sta- 
tionary orbit (or random fixed point) for a random dynamical system $\phi$ if

$$
\phi(t, \omega, y(\omega))=y\left(\theta_{t} \omega\right), \quad \text { a.s., for } t \in \mathbb{R}^{+}, \omega \in \Omega \text {. }
$$

In the following, we present an example to illustrate the abstract definition of stationary orbit.

Example 2. (Stationary orbit for a Langevin equation) Consider a SDE

$$
d X_{t}=-X_{t} d t+d L_{t}^{\alpha}, X_{0}=x .
$$

This SDE defines a random dynamical asystem

$$
\phi(t, \omega, x)=e^{-t} x+\int_{0}^{t} e^{-(t-s)} d L_{s}^{\alpha}(\omega) .
$$

A stationary orbit of this random dynamical system is

$$
y(\omega)=\int_{-\infty}^{0} e^{s} d L_{s}^{\alpha}(\omega)
$$

Indeed, we have $\phi(t, \omega, y(\omega))=y\left(\theta_{t} \omega\right)$, i.e., $y(\omega)=\int_{-\infty}^{0} e^{s} d L_{s}^{\alpha}(\omega)$ is a stationary orbit for the random dynamical system (6.2).

Lemma 1. Under hypothesis (H.1) and (H.2), the following linear stochastic differential equations

$$
\begin{gathered}
d \xi^{\varepsilon}(t)=\frac{1}{\varepsilon} A \xi^{\varepsilon}(t) d t+\frac{\sigma_{1}}{\varepsilon^{\frac{1}{\alpha_{1}}}} d L_{t}^{\alpha_{1}}\left(\omega_{1}\right), \quad \xi(0)=\xi_{0}, \\
d \eta(t)=B \eta(t) d t+\sigma_{2} d L_{t}^{\alpha_{2}}\left(\omega_{2}\right), \quad \eta(0)=\eta_{0} .
\end{gathered}
$$


have càdlàg stationary orbits $\xi^{1, \frac{1}{\varepsilon}}\left(\omega_{1}\right)$ and $\eta\left(\omega_{2}\right)$, respectively.

$$
\left\{\begin{aligned}
\xi^{1, \frac{1}{\varepsilon}}\left(\omega_{1}\right) & =\frac{\sigma_{1}}{\varepsilon^{\frac{1}{\alpha_{1}}}} \int_{-\infty}^{0} e^{\frac{-A s}{\varepsilon}} d L_{s}^{\alpha_{1}}\left(\omega_{1}\right) \\
\eta\left(\omega_{2}\right) & =\sigma_{2} \int_{-\infty}^{0} e^{-B s} d L_{s}^{\alpha_{2}}\left(\omega_{2}\right)
\end{aligned}\right.
$$

Proof. For $\forall t \geq 0$, the SDE (6.5b) has unique càdlàg solution

$$
\varphi\left(t, \omega_{2}, \eta_{0}\right)=e^{B t} \eta_{0}+\sigma_{2} \int_{0}^{t} e^{B(t-s)} d L_{s}^{\alpha_{2}}\left(\omega_{2}\right)
$$

Then we have

$$
\begin{aligned}
\varphi\left(t, \omega_{2}, \eta\left(\omega_{2}\right)\right) & =e^{B t} \eta\left(\omega_{2}\right)+\sigma_{2} \int_{0}^{t} e^{B(t-s)} d L_{s}^{\alpha_{2}}\left(\omega_{2}\right) \\
& =\sigma_{2} e^{B t} \int_{-\infty}^{0} e^{-B s} d L_{s}^{\alpha_{2}}\left(\omega_{2}\right)+\sigma_{2} \int_{0}^{t} e^{B(t-s)} d L_{s}^{\alpha_{2}}\left(\omega_{2}\right) \\
& =\sigma_{2} \int_{-\infty}^{t} e^{B(t-s)} d L_{s}^{\alpha_{2}}\left(\omega_{2}\right) .
\end{aligned}
$$

On the other hand,

$$
\begin{aligned}
\eta\left(\theta_{t}^{2} \omega_{2}\right) & =\sigma_{2} \int_{-\infty}^{0} e^{-B s} d L_{s}^{\alpha_{2}}\left(\theta_{t}^{2} \omega_{2}\right) \\
& =\sigma_{2} \int_{-\infty}^{0} e^{-B s} d\left(L_{t+s}^{\alpha_{2}}\left(\omega_{2}\right)-L_{t}^{\alpha_{2}}\left(\omega_{2}\right)\right) \quad \text { a.s. } \\
& =\sigma_{2} \int_{-\infty}^{0} e^{-B s} d L_{t+s}^{\alpha_{2}}\left(\omega_{2}\right) \quad \text { a.s. } \\
& =\sigma_{2} \int_{-\infty}^{t} e^{B(t-s)} d L_{s}^{\alpha_{2}}\left(\omega_{2}\right) \\
& =\varphi\left(t, \omega_{2}, \eta\left(\omega_{2}\right)\right) .
\end{aligned}
$$

Thus $\eta\left(\omega_{2}\right)$ is a stationary orbit for a random dynamical system $\varphi$. By the same way, we have $\xi^{1, \frac{1}{\varepsilon}}\left(\omega_{1}\right)$ is a stationary orbit for a random dynamical system generated by (6.5a).

Remark 5. Here we emphasize " - B" has no eigenvalue on the imaginary 
axis.

Remark 6. The process $\left(t, \omega_{1}\right) \rightarrow \xi^{1, \frac{1}{\varepsilon}}\left(\theta_{\varepsilon t}^{1} \omega_{1}\right)$ has the same distribution as the process $\left(t, \omega_{1}\right) \rightarrow \xi^{1,1}\left(\theta_{t}^{1} \omega_{1}\right)$ by the scale property of $\alpha$ - stable process.

Secondly, we provides some important pathwise properties for Lévy process with two-sided time $t \in \mathbb{R}$, which comes from [21, Lemma 1]

Lemma 2. (pathwise boundedness and convergence)

Let $L_{t}$ be a two-sided Lévy process on $\mathbb{R}^{n}$ for which $\mathbb{E}\left|L_{1}\right|<\infty$ and $\mathbb{E}\left|L_{1}\right|=M$. Then we have the following

(1) $\lim _{t \rightarrow \pm \infty}(1 / t) L_{t}=M$, a.s.;

(2) The integrals $\int_{-\infty}^{t} e^{-\lambda(t-s)} d L_{s}(\omega)$ are bounded in $\lambda \geq 1$ on finite time intervals $\left[T_{1}, T_{2}\right]$.

In the following, we will give an example to illustrate the second conclusion of Lemma 1.

Example 3. The integral $\int_{-\infty}^{t} e^{-(t-s)} d B_{s}(\omega)$ is bounded on finite time intervals $[-1,1]$

Proof. Here we take $\lambda=1$, by the Itô's isometry formula, then we have

$$
\begin{aligned}
\mathbb{E}\left(\int_{-\infty}^{t} e^{-(t-s)} d B_{s}(\omega)\right)^{2} & =\int_{-\infty}^{t} e^{-2(t-s)} d s \\
& :=\frac{1}{2} e^{-2 t}
\end{aligned}
$$

Obviously, $\frac{1}{2} e^{-2 t}$ is a continuous function about $t$, it is bounded on finite time intervals $\left[T_{1}, T_{2}\right]$. Therefore the integral $\int_{-\infty}^{t} e^{-(t-s)} d B_{s}(\omega)$ is bounded on finite time intervals $[-1,1]$.

In fact, we have to find a modification of Lévy process $L_{t}$, such that the cocycle property is satisfied for every $\omega \in \Omega$. The following lemma covers the perfection problem for all processes of this work (see [12]). 
Lemma 3. Let $H$ be a separable Banach space, $\left(S_{t}\right)_{t \in \mathbb{R}}$ is a $H$-valued and $\mathcal{F}$ measurable stochastic process with cádlág paths generating a crude cocycle with respect to the metric dynamical system $\left(\Omega, \mathcal{F}, \mathbb{P},\left(\theta_{t}\right)_{t \in \mathbb{R}}\right)$, i.e., for all $t \in \mathbb{R}$ we have

$$
S_{t}=S_{0} \circ \theta_{t}, \quad \mathbb{P}-a . s .
$$

Then there is an $H$-valued process $\hat{S}=\left(\hat{S}_{t}\right)_{t \in \mathbb{R}}$, such that:

(i) The process $S$ and $\hat{S}$ are undistinguishable;

(ii) The process $\hat{S}$ is strictly stationary, i.e.

$$
\hat{S}_{t}(\omega)=\hat{S}_{0}\left(\theta_{t} \omega\right)
$$

for all $t \in \mathbb{R}, \omega \in \Omega$.

In the following, we will present an example to explain why don't we take $\hat{S}_{t}=S_{t} ?$

Example 4. Let $(\Omega, \mathscr{F})=(\mathbb{R}, \mathcal{B}(\mathbb{R}))$ and $\mathbb{P}$ be a probability measure which is equivalent to the Lebesgue-measure and $\theta_{t}(\omega)=\omega+t$. Now we define

$$
\varphi(t, \omega):= \begin{cases}\frac{\sin \left(\theta_{t}(\omega)\right)}{\sin (\omega)}, & \sin (\omega) \neq 0 \\ 1, & \sin (\omega)=0\end{cases}
$$

then $\varphi(0, \omega)=1$ for each $\omega \in \Omega$, and $\varphi$ forms a measurable crude multiplicative cocycle. Indeed, fix $s \in \mathbb{R}$ and $\omega \in \Omega \backslash\{(\pi \mathbb{Z} \cup(\pi \mathbb{Z}-s))\}$, we get

$$
\varphi(t+s, \omega)=\frac{\sin (\omega+t+s)}{\sin (\omega+s)} \cdot \frac{\sin (\omega+s)}{\sin (\omega)}=\varphi\left(t, \theta_{s} \omega\right) \varphi(s, \omega)
$$

for each $t \in \mathbb{R}$.

Now, we set $\omega \in(0, \pi), s=\pi-\omega$ and $t:=\frac{\pi}{2}$, which implies $\varphi(s, \omega)=0$ and $\varphi(t+s, \omega) \neq 0$. In this case, there is no perfect cocycle $\psi$ which is still indistinguishable form $\varphi$. if we assume that $\varphi(\cdot, \omega)=\psi(\cdot, \omega)$ for each $\omega \in \Omega_{1} \subset$ 
$\Omega$, then

$$
\varphi(t+s, \omega)=\psi(t+s, \omega)=\psi\left(t, \theta_{s} \omega\right) \psi(s, \omega)=\psi\left(t, \theta_{s} \omega\right) \varphi(s, \omega)=0
$$

for each $t \in \mathbb{R}$ and $\omega \in \Omega_{1}$.

Since $\varphi(t+s, \omega) \neq 0$ for $t=\frac{\pi}{2}$ and $\omega \in(0, \phi)$, then we have

$$
\begin{array}{r}
(0, \pi) \cap \Omega_{1}=\emptyset, \\
\mathbb{P}\left(\Omega_{1}\right)<1,
\end{array}
$$

which implies that there is no indistinguishable perfect cocycle for $\varphi$.

In the next, we examine the following nonstandard boundary value problem, for any $0 \leq t \leq T$,

$$
\left\{\begin{array}{l}
\frac{d \bar{X}^{\varepsilon}(t)}{d t}=A \bar{X}^{\varepsilon}(t)+F\left(\bar{X}^{\varepsilon}(t)+\xi^{1,1}\left(\theta_{t}^{1} \omega_{1}\right), \bar{Y}^{\varepsilon}(t)+\eta\left(\theta_{\varepsilon t}^{2} \omega_{2}\right)\right), \bar{X}^{\varepsilon}(0)=\gamma\left(\bar{Y}^{\varepsilon}(0)\right), \\
\frac{d \bar{Y}^{\varepsilon}(t)}{d t}=\varepsilon B \bar{Y}^{\varepsilon}(t)+\varepsilon G\left(\bar{X}^{\varepsilon}(t)+\xi^{1,1}\left(\theta_{t}^{1} \omega_{1}\right), \bar{Y}^{\varepsilon}(t)+\eta\left(\theta_{\varepsilon t}^{2} \omega_{2}\right)\right), \bar{Y}^{\varepsilon}(T)=\tilde{y},
\end{array}\right.
$$

where $\varepsilon$ is a small positive parameter, $\tilde{y} \in \mathbb{R}^{m}, T>0$ and $\gamma \in \operatorname{Lip}\left(\mathbb{R}^{m}, \mathbb{R}^{n}\right)$ are given.

Lemma 4. Assume that the hypotheses (H.1)-(H.4) hold. Then for any $\tilde{y} \in$ $\mathbb{R}^{m}, T>0, \gamma \in \operatorname{Lip}\left(\mathbb{R}^{m}, \mathbb{R}^{n}\right)$, there exists a sufficient small positive number $\delta$, such that for $0<\varepsilon<\delta$, the random dynamical system defined by (6.17) has a unique solution $\left(\bar{X}^{\varepsilon}(t, \omega, T, \gamma, \tilde{y}), \bar{Y}^{\varepsilon}(t, \omega, T, \gamma, \tilde{y})\right)$.

Proof. Let $V_{A}^{\varepsilon}(t, \omega)$ and $V_{\varepsilon B}^{\varepsilon}(t, \omega)$ be the fundamental solution of the linear system

$$
\left\{\begin{array}{l}
\frac{d \bar{X}^{\varepsilon}(t)}{d t}=A \bar{X}^{\varepsilon}(t) \\
\frac{d \bar{Y}^{\varepsilon}(t)}{d t}=\varepsilon B \bar{Y}^{\varepsilon}(t)
\end{array}\right.
$$

Then the boundary value problem (6.17) is equivalent to the system of integral 
equations

$$
\left\{\begin{array}{l}
\bar{X}^{\varepsilon}(t)=V_{A}^{\varepsilon}(t) \gamma\left(\bar{Y}^{\varepsilon}(0)\right)+\int_{0}^{t} V_{A}^{\varepsilon}(t-s) F\left(\theta_{s}^{\varepsilon} \omega, \bar{X}^{\varepsilon}(s), \bar{Y}^{\varepsilon}(s)\right) d s \\
\bar{Y}^{\varepsilon}(t)=V_{\varepsilon B}^{\varepsilon}(t-T) \tilde{y}+\varepsilon \int_{T}^{t} V_{\varepsilon B}^{\varepsilon}(t-s) G\left(\theta_{s}^{\varepsilon} \omega, \bar{X}^{\varepsilon}(s), \bar{Y}^{\varepsilon}(s)\right) d s .
\end{array}\right.
$$

To study (6.19), we introduce the following spaces

$$
\mathcal{C}_{1}:=C\left([0, T], \mathbb{R}^{n}\right), \quad \mathcal{C}_{2}:=C\left([0, T], \mathbb{R}^{m}\right)
$$

and endow these spaces with the following norms

$$
\begin{aligned}
\|x\|_{1, \beta} & :=\max _{0 \leq t \leq T} e^{-\beta(T-t)}|x(t)|, \quad \text { for } x \in \mathcal{C}_{1}, \\
\|y\|_{2, \beta} & :=\max _{0 \leq t \leq T} e^{-\beta(T-t)}|y(t)|, \quad \text { for } y \in \mathcal{C}_{2} .
\end{aligned}
$$

Let $\mathcal{C}$ be the product space $\mathcal{C}:=\mathcal{C}_{1} \times \mathcal{C}_{2}, z=(x, y) \in \mathcal{C}$. $\mathcal{C}$ equipped with the norm

$$
\|z\|_{\beta}:=\|x\|_{1, \beta}+\|y\|_{2, \beta},
$$

is a Banach space.

Introduce the following two operators $\mathcal{J}_{1}^{\varepsilon}: \mathcal{C} \rightarrow \mathcal{C}_{1}$ and $\mathcal{J}_{2}^{\varepsilon}: \mathcal{C} \rightarrow \mathcal{C}_{2}$ by

$$
\begin{aligned}
& \tilde{x}(t)=\mathcal{J}_{1}^{\varepsilon}(z(\cdot))[t]:=V_{A}^{\varepsilon}(t) \gamma\left(\bar{Y}^{\varepsilon}(0)\right)+\int_{0}^{t} V_{A}^{\varepsilon}(t-s) F\left(\theta_{s}^{\varepsilon} \omega, \bar{X}^{\varepsilon}(s), \bar{Y}^{\varepsilon}(s)\right) d s, \\
& \tilde{y}(t)=\mathcal{J}_{2}^{\varepsilon}(z(\cdot))[t]:=V_{\varepsilon B}^{\varepsilon}(t-T) \tilde{y}+\varepsilon \int_{T}^{t} V_{\varepsilon B}^{\varepsilon}(t-s) G\left(\theta_{s}^{\varepsilon} \omega, \bar{X}^{\varepsilon}(s), \bar{Y}^{\varepsilon}(s)\right) d s .
\end{aligned}
$$

Define the mapping $\mathcal{J}^{\varepsilon}$ given by

$$
\tilde{z}(\cdot)=\mathcal{J}^{\varepsilon}(z(\cdot))=\left(\begin{array}{c}
\mathcal{J}_{1}^{\varepsilon}(z(\cdot))[t] \\
\mathcal{J}_{2}^{\varepsilon}(z(\cdot))[t]
\end{array}\right) .
$$

It is obvious that a fixed point $z^{\varepsilon}$ of $\mathcal{J}^{\varepsilon}$ represents a solution of the boundary value problem (6.17). Under hypothesis H.1-H.3, $\mathcal{J}^{\varepsilon}$ maps $\mathcal{C}$ into itself, and 
there are constants $a_{1} \geq 1$ and $a_{2} \geq 1$ such that

$$
\begin{gathered}
\left\|V_{A}^{\varepsilon}(t-s)\right\| \leq a_{1} e^{-M_{A}(t-s)}, \text { for } t \geq s, \\
\left\|V_{\varepsilon B}^{\varepsilon}(t-s)\right\| \leq a_{2} e^{\varepsilon\|B\||t-s|}, \text { for any } t, s .
\end{gathered}
$$

In the following, we show that $\mathcal{J}^{\varepsilon}$ is also strictly contractive. Let

$$
\triangle \tilde{x}:=\tilde{x}_{1}-\tilde{x}_{2}, \quad \triangle \tilde{y}:=\tilde{y}_{1}-\tilde{y}_{2}, \quad|\triangle \tilde{z}|:=|\triangle \tilde{x}|+|\triangle \tilde{y}| .
$$

Let $\left(\tilde{x}_{1}(t), \tilde{y}_{1}(t)\right)$ and $\left(\tilde{x}_{2}(t), \tilde{y}_{2}(t)\right)$ satisfy the equations (6.19). Then we have

$$
\begin{aligned}
|\triangle \tilde{x}(t)| & =\mid V_{A}^{\varepsilon}(t) \gamma\left(\tilde{y}_{1}^{\varepsilon}(0)\right)+\int_{0}^{t} V_{A}^{\varepsilon}(t-s) F\left(\theta_{s}^{\varepsilon} \omega, \tilde{x}_{1}^{\varepsilon}(s), \tilde{y}_{1}^{\varepsilon}(s)\right) d s . \\
& -V_{A}^{\varepsilon}(t) \gamma\left(\tilde{y}_{2}^{\varepsilon}(0)\right)-\int_{0}^{t} V_{A}^{\varepsilon}(t-s) F\left(\theta_{s}^{\varepsilon} \omega, \tilde{x}_{2}^{\varepsilon}(s), \tilde{y}_{2}^{\varepsilon}(s)\right) d s \mid \\
& \leq a_{1} e^{-M_{A} t}|| \gamma \|_{L i p}\left|\triangle \tilde{y}^{\varepsilon}(0)\right|+a_{1} C \int_{0}^{t} e^{-M_{A}(t-s)}|\triangle \tilde{z}(s)| d s \\
& \leq a_{1} e^{-M_{A} t}|| \gamma\left\|_{L i p}\left|\triangle \tilde{y}^{\varepsilon}(0)\right|+a_{1} C\right\| \triangle \tilde{z} \|_{\beta} e^{-M_{A} t} e^{\beta T} \int_{0}^{t} e^{\left(M_{A}-\beta\right) s} d s \\
& \leq a_{1} e^{-M_{A} t}|| \gamma\left\|_{L i p}\left|\triangle \tilde{y}^{\varepsilon}(0)\right|+\frac{a_{1} C e^{\beta(T-t)}}{M_{A}-\beta}\right\| \triangle \tilde{z} \|_{\beta} .
\end{aligned}
$$

On the other hand, we get

$$
\begin{aligned}
|\triangle \tilde{y}(t)| & =\varepsilon\left|\int_{T}^{t} V_{\varepsilon B}^{\varepsilon}(t-s) G\left(\theta_{s}^{\varepsilon} \omega, \tilde{x}_{1}^{\varepsilon}(s), \tilde{y}_{1}^{\varepsilon}(s)\right) d s-\int_{T}^{t} V_{\varepsilon B}^{\varepsilon}(t-s) G\left(\theta_{s}^{\varepsilon} \omega, \tilde{x}_{2}^{\varepsilon}(s), \tilde{y}_{2}^{\varepsilon}(s)\right) d s\right| \\
& \leq \varepsilon a_{2} C \int_{T}^{t} e^{\varepsilon\|B\|(t-s)}|\triangle z(s)| d s \\
& \leq \varepsilon a_{2} C\|\triangle z\|_{\beta} e^{\beta T-\varepsilon\|B\| t} \int_{t}^{T} e^{-(\beta-\varepsilon\|B\|) s} d s .
\end{aligned}
$$

We assume $\varepsilon$ to be sufficient small such that

$$
\beta-\varepsilon\|B\|>0
$$


Then, we get

$$
\|\triangle \tilde{y}(t)\|_{2, \beta} \leq \frac{\varepsilon a_{2} C}{\beta-\varepsilon\|B\|}\|\triangle z\|_{\beta} .
$$

Bring (6.30) into (6.27), we get

$$
\|\triangle \tilde{x}\|_{1, \beta} \leq\left(\frac{\varepsilon a_{1} a_{2}\|\gamma\|_{L i p} C}{\beta-\varepsilon\|B\|}+\frac{a_{1} C}{M_{A}-\beta}\right)\|\triangle z\|_{\beta}
$$

Combining (6.31) with (6.30), we have

$$
\|\triangle \tilde{z}\|_{\beta} \leq \rho(\varepsilon)\|\triangle z\|_{\beta}
$$

where

$$
\rho(\varepsilon)=\frac{\varepsilon a_{2} C}{\beta-\varepsilon\|B\|}\left(1+a_{1}\|\gamma\|_{L i p}\right)+\frac{a_{1} C}{M_{A}-\beta} .
$$

Note that,

$$
\rho^{\prime}(\varepsilon)=\frac{a_{2} C \beta}{(\beta-\varepsilon\|B\|)^{2}}\left(1+a_{1}\|\gamma\|_{L i p}\right)>0, \quad \rho(0)<1 .
$$

Then there is a sufficiently small constant $\delta>0$ and a constant $\rho_{0} \in(0,1)$, such that

$$
0<\rho(\varepsilon) \leq \rho_{0}<1, \text { for } \varepsilon \in(0, \delta),
$$

which implies that $\mathcal{J}^{\varepsilon}$ is strictly contractive. Therefore the system (6.17) has a unique solution $\left(\bar{X}^{\varepsilon}(t, \omega, T, \gamma, \tilde{y}), \bar{Y}^{\varepsilon}(t, \omega, T, \gamma, \tilde{y})\right)$.

Remark 7. There is a one-to-one relation between $\tilde{y}$ and $\bar{Y}^{\varepsilon}(0, \omega, T, \gamma, \tilde{y})=y_{0}$. Since the operator $\mathcal{J}$ explicitly depends on $\gamma$, we use in the following the notation $\mathcal{J}_{\gamma}^{\varepsilon}$. Moreover, for any $\gamma \in \mathcal{L}$ and $t=T$, the map

$$
\mathbb{R}^{m} \ni y_{0} \rightarrow \phi_{2}^{\varepsilon}\left(t, \theta_{-t}^{\varepsilon},\left(\gamma\left(y_{0}\right), y_{0}\right)\right)=\tilde{y} \in \mathbb{R}^{m}
$$

is invertible, and the inverse mapping $\xi^{\varepsilon}$ is given by

$$
\xi^{\varepsilon}\left(T, \theta_{T}^{\varepsilon} \omega, \gamma\right)(\tilde{y})=\bar{Y}^{\varepsilon}(0, \omega, T, \gamma, \tilde{y})
$$


In the following lemma, we will illustrate the path property and dependence on the function $\gamma$.

Lemma 5. Assume that the hypotheses (H.1)-(H.4) hold. Then for given $\omega \in \Omega, T>0, \tilde{y} \in \mathbb{R}^{m}, t \in[0, T]$ and for sufficiently small $\varepsilon$, the solution $z^{\varepsilon}(t, \omega, T, \gamma, \tilde{y})$ of (6.17) depends Lipschitz continuously on $\gamma$.

$$
\sup _{\tilde{y} \in \mathbb{R}^{m}}\left\|z^{\varepsilon}\left(\cdot, \omega, T, \gamma_{1}, \tilde{y}\right)-z^{\varepsilon}\left(\cdot, \omega, T, \gamma_{2}, \tilde{y}\right)\right\|_{\beta} \leq \frac{a e^{-\beta T}}{1-\rho(\varepsilon)}\left\|\gamma_{1}-\gamma_{2}\right\|_{\infty} .
$$

Proof. For $\gamma_{1}$ and $\gamma_{2}$ any functions in $\mathcal{L}$, define

$$
\triangle_{\gamma} z^{\varepsilon}(t):=z^{\varepsilon}\left(t, \omega, T, \gamma_{1}, \tilde{y}\right)-z^{\varepsilon}\left(t, \omega, T, \gamma_{2}, \tilde{y}\right) .
$$

By Lemma 4 with fixed $(\omega, T, \gamma, \tilde{y})$, there exists a unique fixed point $z^{\varepsilon}$ of the operator $\mathcal{J}_{\gamma}^{\varepsilon}$. Thus, we get

$$
\begin{aligned}
\left\|\triangle_{\gamma} z^{\varepsilon}\right\|_{\beta} & =\left\|\mathcal{J}_{\gamma_{1}}^{\varepsilon} z^{\varepsilon}\left(\cdot, \omega, T, \gamma_{1}, \tilde{y}\right)-\mathcal{J}_{\gamma_{2}}^{\varepsilon} z^{\varepsilon}\left(\cdot, \omega, T, \gamma_{2}, \tilde{y}\right)\right\|_{\beta} \\
& \leq\left\|\mathcal{J}_{\gamma_{1}}^{\varepsilon} z^{\varepsilon}\left(\cdot, \omega, T, \gamma_{1}, \tilde{y}\right)-\mathcal{J}_{\gamma_{1}}^{\varepsilon} z^{\varepsilon}\left(\cdot, \omega, T, \gamma_{2}, \tilde{y}\right)\right\|_{\beta} \\
& +\left\|\mathcal{J}_{\gamma_{1}}^{\varepsilon} z^{\varepsilon}\left(\cdot, \omega, T, \gamma_{2}, \tilde{y}\right)-\mathcal{J}_{\gamma_{2}}^{\varepsilon} z^{\varepsilon}\left(\cdot, \omega, T, \gamma_{2}, \tilde{y}\right)\right\|_{\beta} \\
& \leq \rho(\varepsilon)\left\|\triangle_{\gamma} z^{\varepsilon}\right\|_{\beta}+\left\|V_{A}^{\varepsilon}(\cdot, \omega)\right\|_{\beta}\left\|\gamma_{1}-\gamma_{2}\right\|_{\infty} .
\end{aligned}
$$

By hypothesis (H.3), we have

$$
\left\|\triangle_{\gamma} z^{\varepsilon}\right\|_{\alpha} \leq \frac{a_{1} e^{\alpha T}}{1-\rho(\varepsilon)}\left\|\gamma_{1}-\gamma_{2}\right\|_{\infty}
$$

This proof is complete.

Define the random graph transform by

$$
\begin{aligned}
\psi^{\varepsilon}(T, \omega, \gamma)(\tilde{y}) & :=\phi_{1}^{\varepsilon}\left(T, \omega,\left(\gamma\left(\xi^{\varepsilon}\left(T, \gamma_{T}^{\varepsilon} \omega, \gamma\right)(\tilde{y})\right), \xi^{\varepsilon}\left(T, \gamma_{T}^{\varepsilon} \omega, \gamma\right)(\tilde{y})\right)\right. \\
& =\bar{X}^{\varepsilon}(T, \omega, T, \gamma, \tilde{y}) \\
& =V_{A}^{\varepsilon}(T) \gamma\left(\bar{Y}^{\varepsilon}(0)\right)+\int_{0}^{T} V_{A}^{\varepsilon}(t-s) F\left(\theta_{s}^{\varepsilon} \omega, \bar{X}^{\varepsilon}(s), \bar{Y}^{\varepsilon}(s)\right) d s
\end{aligned}
$$


such that the cocycle property is satisfied. By the similar techniques [19, Lemma 4.1] and [19, Lemma 4.7], we get the following two lemmas.

Lemma 6. For $\omega \in \Omega$, let $\gamma^{\varepsilon}(\omega, \cdot) \in \operatorname{Lip}\left(\mathbb{R}^{m}, \mathbb{R}^{n}\right)$. Suppose that for $\gamma^{\varepsilon}(\omega, \cdot)$ a random fixed points of $\psi^{\varepsilon}$, we have

$$
\psi^{\epsilon}\left(t, \omega, \gamma^{\varepsilon}(\omega, \cdot)\right)(\tilde{y})=\gamma^{\varepsilon}\left(\theta_{t}^{\epsilon} \omega, \tilde{y}\right)
$$

for $t>0$ and $\omega \in \Omega$. Then the random Lipschitz manifold defined by

$$
\mathcal{M}^{\epsilon}(\omega):=\left\{\left(\gamma^{\varepsilon}(\omega, \tilde{y}), \tilde{y}\right) \mid \tilde{y} \in \mathbb{R}^{m}\right\}
$$

is positively invariant.

Lemma 7. Assume that the hypotheses (H.1)-(H.4) hold. Then for sufficiently small $\varepsilon$ and sufficiently large $T$, the graph transform $\psi^{\varepsilon}(T, \omega, \cdot)$ maps the set $\mathcal{L}_{\kappa}$ into itself, where $\kappa$ is any positive number satisfying

$$
\kappa \geq \kappa^{*}=\frac{a_{2}}{1-\beta_{0}-a_{1} a_{2} e^{-\frac{T \beta}{2}}},
$$

where $\beta_{0}$ is any given number from the interval $\left(\frac{a_{1} C}{M_{A}-\beta}, 1\right)$.

Set

$$
\mathscr{H}^{\varepsilon}(\omega):=\bigcup_{t \geq 0} \psi^{\varepsilon}\left(t, \theta_{-t}^{\varepsilon} \omega, L_{\kappa^{*}}\right)
$$

Then we have

$$
\psi^{\varepsilon}\left(t, \omega, \mathscr{H}^{\varepsilon}(\omega)\right) \subset \mathscr{H}^{\varepsilon}\left(\theta_{t}^{\varepsilon} \omega\right), \quad t \geq 0,
$$

By a similar technique as in [19, Lemma 4.10], we are able to prove the cocycle property for the graph transform $\psi^{\varepsilon}$. By means of the graph transform $\psi^{\varepsilon}$, we define an operator $\mathcal{A}^{\varepsilon}$ via

$$
(\omega, \tilde{y}) \rightarrow \mathcal{A}^{\varepsilon}(\gamma)(\omega, \tilde{y}):=\psi^{\varepsilon}\left(T, \theta_{-T}^{\varepsilon} \omega, \gamma\left(\theta_{-T}^{\varepsilon} \omega\right)\right)(\tilde{y}) .
$$

Now we prove the exponential tracking property. 
Lemma 8. Assume that the hypotheses (H.1)-(H.4) hold. Then for sufficiently small $\varepsilon$, the Lipschitz invariant manifold has the exponential tracking property in the following sense:

For every solution $\bar{z}^{\varepsilon}(t, \omega)=\left(\bar{x}^{\varepsilon}(t, \omega), \bar{y}^{\varepsilon}(t, \omega)\right)$ for (6.17), there is an orbit $\overline{\bar{z}}^{\varepsilon}(t, \omega)=\left(\overline{\bar{x}}^{\epsilon}(t, \omega), \overline{\bar{y}}^{\epsilon}(t, \omega)\right)$ on the manifold $\mathcal{M}^{\epsilon}(\omega)$ which satisfies the evolutionary equation

$$
\dot{\tilde{y}}^{\varepsilon}=B \tilde{y}^{\varepsilon}+G\left(\gamma^{\varepsilon}\left(\theta_{t}^{\varepsilon} \omega, \tilde{y}^{\varepsilon}\right), \tilde{y}^{\varepsilon}, \theta_{t}^{\varepsilon} \omega\right)
$$

such that

$$
\left\|\bar{z}^{\varepsilon}(t, \omega)-\overline{\bar{z}}^{\varepsilon}(t, \omega)\right\|_{\infty} \leq C_{\tilde{\kappa}, \varepsilon} e^{\frac{-\tilde{\kappa} t}{\varepsilon}}\left\|\bar{z}_{0}-\overline{\bar{z}}_{0}\right\|_{\infty}
$$

with $\bar{z}_{0}=\left(\bar{x}^{\varepsilon}(0), \bar{y}^{\varepsilon}(0)\right), \overline{\bar{z}}_{0}=\left(\overline{\bar{x}}^{\varepsilon}(0), \overline{\bar{y}}^{\varepsilon}(0)\right)$ and some positive constant $\tilde{\kappa}$.

Proof. Thanks to (6.40), we can use dominated convergence theorem and the same method in [20, Theorem 4.2] to obtain the required results.

Acknowledgements. We would like to thank Xianming Liu (Huazhong University of Sciences and Technology, China) for helpful discussions.

\section{Reference}

\section{References}

[1] Bain A, Dan C(2009) Fundamentals of Stochastic Filtering. Springer, New York

[2] Evensen G(2009) Data Assimilation: The Ensemble Kalman Filter. Springer-Verlag, Berlin

[3] Rozovskii, B(1990) Stochastic Evolution System: Linear Theory and Application to nonlinear Filtering. Springer, New York

[4] Ceci C, Colaneri K(2014), The Zakai equation of nonlinear filtering for jumpdiffusion observations: existence and uniqueness. Applied Mathematics and Optimization 69: 47-82 
[5] Qiao H, Duan J(2015) Nonlinear filtering of stochastic dynamical systems with Lévy noises. Adv. Appl. Prob. 47: 902-918

[6] Sun X, Duan J, Li X, Wang X(2015) State estimation under non-Gaussian Lévy noise: A modified Kalman filtering method. Banach Center Publications 105: 239-246

[7] Popa A, Sritharan A(2009) Nonlinear filtering of Itô-Lévy stochastic differential equations with continuous observations. Communications on Stochastic Analysis 3: 313-330

[8] Dror G(2007) Strong convergence rate for two-time-scale jump-diffusion stochastic differential systems. Siam Journal on Multiscale Modeling and Simulation 6: 577-594

[9] Bao J, Yin G, Yuan C(2017) Two-time-scale stochastic partial differential equations driven by $\alpha$-stable noises: Averaging principles. Bernoulli 23: 645669.

[10] Khasminskii R. Z., Yin G(1996) On transition densities of singularly perturbed diffusions with fast and slow components. SIAM J. APPL. MATH. 56: 1794-1819.

[11] Zhang Y, Cheng Z, Zhang X, Chen X, Duan J, Li X(2017), Data assimilation and parameter estimation for a multiscale stochastic system with $\alpha$ -stable Lévy noise. Journal of Statistical Mechanics Theory and Experiment 11: 113401

[12] Kümmel K(2016) On the dynamics of Marcus type stochastic differential equations. Doctoral thesis, Friedrich-Schiller-Universität Jena

[13] Wang W, Roberts A J(2013) Slow manifold and averaging for slow-fast stochastic differential system. Journal of Mathematical Analysis and Applications. 398: 822-839. 
[14] Qiao H, Zhang Y, Duan J(2018) Effective filterings on random slow manifold. Nonlinearity 31 (2018) 4649-4666.

[15] Applebaum D(2009) Lévy Processes and Stochastic Calculus. Cambridge University Press, UK

[16] Duan J(2015) An Introduction to Stochastic Dynamics. Cambridge University Press, New York

[17] Arnold L(1998) Random Dynamical Systems. Springer, Berlin

[18] Lachout P(1992) A Skorohod space of discontinuous functions on a general set. Acta Universitatis Carolinae. Mathematica et Physica 33: 91-97

[19] Schmalfuss B, Schneider K(2008) Invariant manifolds for random dynamical systems with slow and fast variables. Journal of Dynamics and Differential Equations 20: 133-164

[20] Fu H, Liu X, Duan J(2012) Slow manifolds for multi-time-scale stochastic evolutionary systems. Communications in Mathematical Sciences 11: 141162

[21] Liu X, Duan J, Liu J, Kloeden PE(2010) Synchronization of dissipative dynamical systems driven by non-Gaussian Lévy noises. International Journal of Stochastic Analysis 2010: 502803 